\title{
« Troubles dans le local » \\ Migrations transnationales et transformations culturelles à Java
}

Loïs Bastide,

Postdoctorant, Département de Sociologie, Université de Genève.

Thèmes de recherche actuels : catastrophes naturelles et processus de reconstruction sociale en Indonésie ; analyse organisationnelle et communicationnelle de la pandémie H1N1 de 2009 aux Etats-Unis, au Japon, en Suisse et par l'OMS.

Publications : Loïs Bastide (A paraître), « «Migrer, être affecté » Emotions et expériences spatiales entre Java, Kuala Lumpur et Singapour », REMI, 2014 ; Loïs Bastide, « Ethnographie de l'ailleurs et ailleurs ethnographiques : postcolonialité, subjectivation et construction des espaces de l'enquête en Asie du sud-est », dans Laurence Rouleau-Berger (dir.) Sociologie et Cosmopolitisme Méthodologique, PUM, 2012.

\section{Introduction}

Depuis plus de quarante ans, l'Indonésie s'est engagée dans l'exportation de ses travailleurs : d'abord vers les pays du Golfe, à l'époque des chocs pétroliers, puis vers d'autres pays d'Asie alors qu'une croissance très inégale redessinait la région autour de nouveaux centres et de nouvelles marges économiques. Dans ce vaste mouvement régional, les Philippines et l'Indonésie se sont imposés comme les grands pourvoyeurs de main d'œuvre en Asie de l'Est et du Sud-Est. Dans le sillage de l'expérience philippine, l'entrée précoce sur les nouveaux marchés de la main d'œuvre transnationale s'est accompagnée en Indonésie d'un processus d'institutionnalisation progressive qui a permis à l'Etat de garder la main sur ces circulations, avec la création d'agences publiques chargées de réguler les flux et l'ouverture au secteur privé de l'activité de recrutement et de placement. Si les migrations ont cru moins vite que chez le voisin du nord, elles n'en ont pas moins atteint un volume considérable : entre 1969 et 1994 , les contingents annuels de migrants officiellement placés sont passés de 10.000 à $140.000^{1}$. Pour la seule année 2007, le volume excédait les 700.000 travailleurs répartis essentiellement

\footnotetext{
${ }^{1}$ Hugo, « Labour Export from Indonesia », ASEAN Economic Bulletin, 12(2), 1995, p.275-298.
} 
entre l'Asie-Pacifique, le Proche et le Moyen Orient ${ }^{2}$. Ce faisant, ces circulations ont acquis une importance politique croissante.

La dépendance grandissante de l'Etat à l'égard de ces nouvelles mobilités tient essentiellement à deux choses : elle a d'abord un aspect économique puisque le commerce de la main-d'œuvre est devenu un des premiers secteurs d'exportation du pays, avec 7.4 milliards de dollars de « remises » en $2013^{3}$. Elle a aussi un aspect social, dans la mesure où les flux de travailleurs absorbent des populations souvent paupérisées, marginalisées sur le plan domestique, dont les revenus irriguent souvent, par surcroît, des espaces délaissés par les pouvoirs publics. En cela, ces transnationalismes d'inscrivent dans le sillage des grands mouvements de populations planifiés qui ont vu l'Etat administrer des migrations des îles centrales vers les îles périphériques ${ }^{4}$, depuis la colonisation. Ces politiques, outre qu'elles entendaient mettre en valeur des zones reculées de l'archipel, visaient aussi à renforcer la sécurité du territoire (en le peuplant de nationaux) et l'unité nationale, en améliorant le niveau de vie des populations et la répartition territoriale du développement ${ }^{5}$. Les migrations contemporaines rejouent sur une autre scène et à une autre échelle cette politisation des mobilités.

Ces transnationalismes sont ainsi mobilisés comme un instrument politique. Ils emportent aussi, cependant, des transformations profondes et imprévisibles qui bouleversent les conditions et les modes de vie aux quatre coins de l'archipel. Si dans certaines populations les migrations peuvent s'appuyer sur des pratiques circulatoires anciennes qui facilitent leur « domestication» et régulent leur impact, cette culture est beaucoup moins présente à Java, où les populations sont traditionnellement plus sédentaires ${ }^{6}$. Là, ces mobilités altèrent fortement les structures sociales, dans des espaces de plus en plus tributaires des circuits transnationaux.

\footnotetext{
${ }^{2}$ Source : BNP2TKI, cité dans Migration News

${ }^{3}$ Source : Ministère du Travail et de la Transmigration. Encore ces chiffres sont-ils très sous-évalués : une part significative des flux échappe en effet à la recension de par son mode de transfert. Par ailleurs, pour réellement prendre la mesure de l'exportation de main-d'œuvre comme secteur économique, il conviendrait d'ajouter à cela les revenus des acteurs publics et privés du placement.

${ }^{4}$ Daniel Benoit, Pierre Levang et Olivier Sevin, Transmigration et migrations spontanées en Indonésie, IRD Ed., 1989; Rebecca Elmhirst, «Space, identity politics and resource control in Indonesia's transmigration programme », Political Geography, 18(7), 1999, p.813-835; Olivier Sevin, "Que sont devenus les transmigrants? Vingt-cinq ans de transmigration dans le Centre-Kalimantan (Indonésie) », Les Cahiers d'Outre-Mer, 244(4), 2008, p.433-457; Pierre Levang et Olivier Sevin, « 80 ans de Transmigration en Indonésie (1905-1985)», Annales de Géographie, 98(549), 1989, p.538-566 ; Tirtosudarmo, «Demography and security: Transmigration policy in Indonesia», dans Myron Weiner et Sharon S. Russel (dir.), Demography and National Security, Berghahn Book, 2001, p. 199-227

${ }^{5}$ Tirtosudarmo, « Demography and security : Transmigration policy in Indonesia », cite, p.199-227.

${ }^{6}$ Même s'il faut sans doute se garder de surévaluer la valeur culturelle de cette immobilité. Cf: Adrian Vickers, "The country and the cities », dans Kevin Hewison et Ken Young (dir.), Transnational migration and work in Asia, Routledge, pp. 37-56; Graeme J. Hugo, «Forced migration in Indonesia: Historical perspectives », Asian and Pacific migration journal, 15(1), 2006, p. 53-92.
} 
L'enjeu du texte est d'analyser ces tensions, dans le contexte javanais, en posant pour hypothèse : que les circulations migratoires délitent l'ordre social hérité (dans les espaces d'origine) du fait des tensions croissantes entre les formes traditionnelles de l'expérience locale, d'une part, les « épreuves $»^{7}$ vécues, les pratiques et les modes de vie développés sur les routes migratoires, d'autre part. Cette articulation donne lieu à des processus de négociation qui transforment les rapports de force et ouvrent de nouvelles modalités du «faire communauté », " au pays ». Ces processus, soutiendrons-nous, dévoilent la complicité entre les structures traditionnelles de l'expérience communautaire et une certaine organisation des rapports de pouvoir, qui demeurait pour l'essentiel invisible jusqu'à l'irruption des «turbulences migratoires $»^{8}$. Ce faisant, elles ouvrent sur des contestations qui redessinent l'espace du politique sur le plan local.

\section{Introduction au terrain}

L'analyse s'appuie sur une recherche de vingt-deux mois dispersée entre l'Indonésie, la Malaisie et Singapour ${ }^{9}$, organisée sur le modèle de $l^{\prime}$ " ethnographie multisite $»^{10}$. A Java, une année de terrain a été consacrée à l'étude d'un village très inséré sur les circuits migratoires, auprès des « partant » et des « restant », des candidats au départs, des migrants au retour, de familles plus ou moins directement affectées par le «fait migratoire ». A Kuala Lumpur et Singapour nous avons passé deux et six mois à enquêter auprès d'ONG diversement engagées auprès des migrants, et en immersion dans des réseaux de travailleurs indonésiens que nous avons suivis dans leurs activités quotidiennes ${ }^{11}$.

A Java, le terrain s'est situé dans un village côtier du Territoire Spécial de Yogyakarta (DIY), sur la côté sud de Java, petite commune de deux mille cent habitants ${ }^{12}$. Convenons de l'appeler

\footnotetext{
${ }^{7}$ Dans l'ensemble du texte, nous ferons référence à la définition développée par Danilo Martuccelli : l'épreuve est conçue comme «mise à l'épreuve », dans laquelle l'individu "éprouve», simultanément la résistance du social et sa consistance d'individu. L'imposition d'épreuves réglées, qui s'imposent collectivement, permet d'expliquer des formes régulières d'individuation, dans un contexte spécifiques. Danilo Martuccelli, Forgé par l'épreuve: l'individu dans la France contemporaine, Paris, Armand Colin, coll. «Individu et société », 2006.

${ }^{8}$ Geneviève Cortes et Laurent Faret, Les circulations transnationales: lire les turbulences migratoires contemporaines, Paris, Armand Collin, 2009.

${ }^{9}$ Recherche conduite dans le cadre d'une thèse de doctorat en sociologie intitulée « Habiter le transnational. Politiques de l'espace, travail globalisé et subjectivités entre Java, Kuala Lumpur et Singapour », dirigée par Laurence Roulleau-Berger et soutenue le 16 septembre 2011 à l'ENS de Lyon.

${ }^{10}$ Pour une synthèse des tenants méthodologiques, théoriques et épistémologiques de l'ethnographie multisite on pourra consulter: Falzon, Multi-sited ethnography: Theory, praxis and locality in contemporary researche, Ashgate Publishing, 2009

${ }^{11}$ Loïs Bastide, «Ethnographie de l'ailleurs et ailleurs ethnographiques : postcolonialité, subjectivation et construction des espaces de l'enquête en Asie du sud-est », dans Laurence Rouleau-Berger (dir.) Sociologie et Cosmopolitisme Méthodologique, PUM, 2012.

$12 \mathrm{Au}$ recensement de 2008.
} 
Banyu Putih ${ }^{13}$. La municipalité (desa) est située dans la régence ${ }^{14}$ de Kulon Progo, sur un terrain ingrat, sablonneux, dans un climat sec. Elle est aussi la moins bien dotée du sous-district en surface agricole, et la seule à ne pas bénéficier d'un système d'irrigation. La faible productivité des sols, quand on sait que la population vivait essentiellement de l'agriculture jusqu'à la seconde moitié des années 1980, explique en partie que les habitants aient été parmi les premiers à partir quand l'opportunité s'est présentée.

Les migrations de travail ont débuté en 1982 quand cinq jeunes hommes ont été placés en Iraq par le bureau local du ministère des «ressources humaines et de la transmigration » (Menakertrans), suivis en 1984 par un premier groupe de femmes, recrutées en Arabie Saoudite. Les mobilités économiques se sont matérialisées au retour des «pionniers » dans la multiplication de maisons permanentes, des acquisitions de terres et de bétail et l'accès à une meilleure éducation pour les enfants. A une époque où l'habitat était basique, où la plupart des enfants étaient mis au travail, au mieux, dès la fin de l'école élémentaire, elles ont suscité un vif intérêt, inaugurant un phénomène de migration en chaîne. Depuis la seconde moitié des années 1990 les migrations se sont développées, en sorte que 80 à 90\% des familles sont aujourd'hui enrôlées sur ces nouveaux circuits transnationaux.

Le village a donc pris part aux premières vagues des migrations «étatisées », s'imposant rapidement comme un des plus importants bassins de recrutement de la régence et, partant, du $\mathrm{DIY}^{15}$. La prévalence du fait migratoire et l'accumulation consécutive des « savoir-circuler » 16 et du capital économique a permis aux habitants de se diriger progressivement vers les destinations les plus valorisées ou les mieux rémunératrice: alors que la Malaisie est aujourd'hui la seconde destination la plus représentée au niveau national - bien que peu lucrative $^{17}$-, les femmes du village migrent plutôt vers Taiwan, Hong Kong ou l'Arabie Saoudite (où elles espèrent pouvoir faire le petit ou le grand pèlerinage à la Mecque) ; les

\footnotetext{
${ }^{13}$ Les noms et les toponymes ont été modifiés.

${ }^{14}$ L'administration territoriale en zone rurale se décline comme suit en Indonésie : province, régence (kabupaten) - héritée du système d'administration colonial -, kecamatan (sorte de communauté de commune ou de " sous district » à l'américaine), village (desa ou kelurahan hameau (dusun ou pedukuhan).

${ }^{15}$ Pendant la décennie 1980-1990 Kulon Progo représentait 23\% de l'émigration provinciale contre 6\% pour Sleman, et 16\% pour Bantul, les deux autres régences du Territoire Spécial (Sevin, Migrations, colonisation agricole et terres neuves en Indonésie, 1:411). En 2007 le territoire représentait $65 \%$ des départs de migrants TKI enregistrés dans le DIY et $98 \%$ des placements officiels en Malaysia (BPS, Daerah Istimewa Yogyakarta dalam angka 2008). Ces chiffres frappent d'autant qu'il convient de les rapporter à la faible densité démographique de la régence par rapport à Bantul et Sleman. Il faut cependant les prendre avec prudence dans la mesure où la majorité des migrants échappent à la procédure officielle, soit qu'ils n'apparaissent pas du tout dans les statistiques, soit qu'ils soient enregistrés ailleurs - Java-Centre souvent, Jakarta ou Surabaya, les grands points d'embarquement.

${ }^{16}$ Tarrius et Missaoui, Les nouveaux cosmopolitismes: Mobilités, identités, territoires, Ed. de l'Aube, 2000.

${ }^{17}$ Mais qui bénéficie de tarifs de placement relativement bas. Ce qui explique l'attrait de la destination.
} 
hommes, quand ils s'en trouvent en position, se dirigent vers la Corée du Sud, Taiwan, voire le Japon.

Sans surprise ces nouvelles mobilités ont profondément affecté les relations sociales au village. D'abord, conforme en cela à une tendance forte des migrations internationales et à la composition globale des flux migratoires en provenance d'Indonésie, les circulations se féminisent ${ }^{18}$. Il s'en suit que les hommes sont de plus en plus nombreux à assumer le travail reproductif tandis que leurs épouses s'emparent des tâches productives, aujourd'hui délocalisées dans une dizaine de pays d'Asie, du Proche et du Moyen-Orient. Alors que les femmes émergent au centre de la vie économique ${ }^{19}$ la distribution genrée des rôles sociaux, fortement liée à l'intervention biopolitique et idéologique de l'Etat dans la sphère reproductive $^{20}$, est déstabilisée :

«En tant qu'homme, j'ai la responsabilité de pourvoir aux besoins de ma famille. [...]. Alors pour moi, quelle que soient la situation il est inutile que ma femme reparte travailler à l'étranger. L'estime de soi d'un homme dont la femme travaille à l'étranger est affectée. Quoi qu'il arrive, je me dois d'être responsable, et il vaut mieux que ma femme reste à la maison ».

Comme pour Cecep, qui a élevé seul ses deux enfants durant près d'une décennie pendant que Tya, sa femme, travaillait en Arabie Saoudite puis à Taiwan, ce trouble est vécu souvent dans les termes d'une atteinte à la virilité. Ces recompositions suscitent ainsi de fortes incertitudes identitaires, particulièrement chez les hommes, tiraillés entre les attentes liées à leur statut traditionnel au sein de la société et de la famille, et leur marginalisation au sein de l'économie familiale et locale.

Les stratifications sociales ${ }^{21}$ sont aussi fragilisées par des mobilités économiques rapides : les migrants en Corée du Sud, à Hong Kong ou à Taiwan font valoir des salaires mensuels qui oscillent entre quatre et vingt millions de rupiah ${ }^{22}$ quand les revenus des travailleurs, dans la régence, se situaient en 2008, autour de cinq cent mille rupiahs, souvent moins. Par-delà ces écarts, les effets de ces mobilités sur le collectif villageois tiennent surtout à leur distribution

\footnotetext{
${ }^{18}$ Entre 2006 et 2012, 3,048 millions de femmes indonésiennes ont été officiellement placées à l'étranger, contre 950 mille hommes (BNP2TKI2013).

${ }^{19}$ Sur ce thème voir aussi: Rebecca Elmhirst, « Tigers and Gangsters », Population, Space and Place, 13(3), 2007, p.225-238; Rachel Silvey, « Geographies of Gender and Migration: Spatializing Social Difference », International Migration Review, 40(1), 2006, p. 63-81.

20 Brenner, The Domestication of Desire; Robinson, Gender, Islam, and democracy in Indonesia, Princeton, Prinston University Press, 1998.

${ }^{21}$ Notre lecture des processus de stratifications sociale est d'inspiration weberienne : ce cadre théorique permet en effet de traiter aussi, à côté de la classe sociale, la question du statut et du prestige social, particulièrement significative à Java.

${ }_{22}$ En 2008-2009, au moment des terrains, un euro équivalait approximativement à 14000 rupiah.
} 
sociale. Car dans les conditions de pauvreté qui prévalaient à la fin des années 1970 les édiles villageois, recrutés dans les rangs de la petite aristocratie traditionnelle ${ }^{23}$ étaient relativement privilégiés, cumulant souvent les statuts de fonctionnaires municipaux et de petits propriétaires terriens, jouissant en outre de l'usage des apanages communaux - tanah bengkok - attachés à leur fonction.

De manière prévisible les premiers à saisir l'opportunité d'un départ ont été recrutés parmi les plus pauvres, chez ceux pour qui le besoin de mobilité économique était le plus urgent. La petite aristocratie municipale, qui n'était pas soumise à la même pression, est demeurée beaucoup plus sédentaire. Du fait de l'homologie entre hiérarchies symboliques et structures de classes, des «économies morales » ${ }^{24}$ ont émergé, liées à ces nouvelles pratiques circulatoires : au sein de ces répertoires de normes, de valeurs, de sentiments moraux et de jugements encore instables, le prestige social s'est progressivement attaché à l'immobilité tandis que la migration, parce qu'elle concernait d'abord les segments les plus marginalisés de la société villageoise - les femmes et, parmi elles, les plus jeunes et les plus pauvres -, s'est construite comme une «épreuve » à l'issue au mieux ambivalente, susceptible de miner le statut social des acteurs.

Cette distribution des biens symboliques a produit de nouveaux types d'inégalités, liés à la distribution de la « reconnaissance sociale ${ }^{25}$, qui ont participé à renforcer la sédentarité des notables. Dans le même temps, le volume des «remises» en provenance des espaces de migration contribuait à renverser les hiérarchies économiques, creusant en vingt ans un écart croissant entre l'ordre symbolique et l'ordre du pouvoir économique (la « classe », au sens de Weber).

Enfin, les migrations corrodent l'ordre des rapports intergénérationnels. Car à l'heure où, bien souvent, la troisième génération de migrants part dès la fin du lycée, libre encore des liens matrimoniaux, certains peinent à se réinscrire au retour dans la vie collective, son maillage serré de prescriptions sociales, ses régimes de réciprocité et d'obligation. C'est particulièrement vrai pour ceux qui ont été exposés à la pluralité des normes, des pratiques et

\footnotetext{
${ }^{23}$ Hans-Dieter Evers, «The Bureaucratization of Southeast Asia », Comparative Studies in Society and History, 29(4), 1987, pp. 666-685.

${ }^{24}$ Plutôt qu'aux usages de Thompson ou J. Scott nous adoptons la conception plus large de l'économie morale proposée par D. Fassin. Plutôt que de restreindre son usage à l'étude des situations de domination et de résistance, il propose de la voir : «[...] comme la production, la répartition, la circulation et l'utilisation de sentiments moraux, des émotions et des valeurs, des normes et des obligations dans l'espace social 》. Didier FASSIN, «Les économies morales revisitées », Annales. Histoire, Sciences Sociales, 64(6), 2009, p. 1257.

${ }^{25}$ Axel Honneth, La lutte pour la reconnaissance, Ed. du Cerf, 2000.
} 
des « épreuves » dans les grandes métropoles régionales qui peinent à accepter le retour à une position subalterne et figée dans l'ordre générationnel des positions. Sans surprise, ce phénomène affecte particulièrement les femmes : c'est qu'au moment de se réinstaller, elles sont confrontées aux effets de leur éloignement des dispositifs collectifs de contrôle social, pendant leur migration. Celles qui vivaient en ville subissent une suspicion particulière, liée à l'impossibilité de contrôler leurs conduites dans un contexte urbain conçu comme particulièrement corrupteur. Pour neutraliser le stigmate social associé à la circulation, les femmes ont alors à faire valoir un fort degré de conformité sociale (particulièrement au regard de la distribution traditionnelle des rôles sexués).

Les inégalités d'accès à la « reconnaissance sociale » et aux biens symboliques se déclinent ainsi selon la classe, le genre et les générations : L'affluence nouvelle de la « roture » et les difficultés à convertir la richesse en prestige social créent des tensions. Les plus jeunes, encore dans leur vingtaine, sont réputés plus turbulents (bandel) que leurs ainés, moins travailleurs et plus difficile à réintégrer dans le collectif. En l'absence de tout programme public d'accompagnement au retour ${ }^{26}$ qui faciliterait ce passage, les jeunes hommes se voient accorder une période d'accommodation pendant laquelle on tolère désormais des écarts de comportement. Cette période de transition est interdite aux femmes. Certaines préfèrent alors rompre définitivement leurs attaches au village, choisissant l'exit face à l'impossibilité de faire voix à des aspirations nouvelles et au refus de faire valoir leur loyautée ${ }^{27}$ à l'égard d'un ordre social qui apparaît désormais injuste et coercitif. Les modes de vie hérités, les hiérarchies sociales et symboliques établies sont ainsi soumis à une pression croissante.

\section{L'économie morale des migrations}

On voit ainsi émerger de nouvelles «économies morales», morcelées et parcellaires, connectées à ces nouvelles « épreuves » sociales. Elles participent à la domestication collective des expériences circulatoires et à la régulation de leurs effets sur les structures sociales en encadrant la négociation de significations partagées et la normalisation de ces nouvelles pratiques. Cependant, ces « économies morales » sont loin de faire consensus : elles semblent

\footnotetext{
${ }^{26}$ On assiste aujourd'hui à des tentatives du gouvernement pour former les migrants au retour à l'entrepreneuriat. Elles demeurent pour l'instant très circonscrites.

${ }^{27}$ Hirschman, Exit, voice, and loyalty, Cambridge, London, Harvard University Press, 1970.
} 
ouvrir plutôt de nouveaux espaces de controverse. Ce faisant, elles apparaissent fragmentées, voire antagoniques.

Alors que les populations demeuraient très sédentaires jusqu'aux années 1980, la migration émerge de plus en plus aujourd'hui comme une étape attendue des trajectoires biographiques, au moment où la troisième génération de migrants s'engage sur les routes migratoires. Mais cette norme émergente est ambivalente, pour autant que la valeur sociale de la circulation demeure ambiguë. Pour les femmes, ses conséquences sont clairement négatives, on l'a vu, qui minent leur statut. Reste que l'éviction hors des routes migratoires est aujourd'hui vécue sur le mode de l'exclusion sociale et devient la première cause de marginalisation économique. Tumina, une jeune femme du village, sédentarisée par la naissance de son fils, ne dit pas autre et exprime l'inconfort de cette condition, quand la circulation devient la norme :

«Quand on me demande... les gens du coin partent tous... : "Ça alors! Tu ne repars pas? Alors je dis "plus tard, quand mon fils sera plus grand». Le problème c'est que dans le coin presque tout le monde part. Alors quand il y a des gens à qui ça déplait [sa sédentarité] je leur dis : "plus tard, quand mon fils sera grand je repartirai. »".

Il serait imprudent néanmoins de traiter ces orientations apparemment paradoxales comme symptômes de désordre ou d'anomie ; elles reflètent plutôt des mutations normatives en cours, encore faiblement institutionnalisées. En d'autres termes, elles invitent à penser ces normativités émergentes et les tensions qui les travaillent comme processus, en les considérant comme autant d'indices de l'accrétion progressive de nouveaux répertoires normatifs et symptômes des résistances qu'elle entraîne.

Ces incertitudes et ce flottement, observables à l'échelle du village dans ces lignes de fracture morales et ces significations discordantes, révèlent des tensions entre les formes héritées de la vie communale, d'une part, et des ordres sociaux émergents, d'autre part, constitués au fil des « épreuves » transnationales, dans la répétition des cycles migratoires. Et il est alors tout sauf anodin que le processus de valorisation symbolique de l'expérience migratoire, en tant qu' « épreuve » collective, apparaisse sous un jour très contrasté quand on l'observe depuis les lieux de migration. En circulant entre différents sites sur les routes migratoires, de nouveaux arrangements sociaux apparaissent en effet à la faveur de l'enquête. D'autres hiérarchies et d'autres « économies morales » se révèlent qui n'appartiennent à aucun lieu en particulier mais 
sont liées, plutôt, à leur articulation dans des parcours individuels et collectifs, c'est-à-dire à l'acte de circuler ${ }^{28}$.

A Kuala Lumpur et Singapour, parmi les femmes qui parviennent à développer des relations sociales et à poser des pratiques hors des espaces de travail ${ }^{29}$, la capacité à circuler et à agencer les routes migratoires au regard des circonstances est une compétence fortement valorisée. $\mathrm{Au}$ village en revanche la « reconnaissance sociale » est davantage attachée à l'expression d'un fort enracinement. A un certain degré, et par comparaison, les hommes font preuve d'une attitude plus conservatrice, au regard notamment des rapports de genre : si les aventures sexuelles forment aujourd'hui un quasi attendu des expériences masculines en migration, beaucoup parmi les travailleurs migrants indonésiens, en Malaysia, s'efforcent de chaperonner leurs compatriotes féminines, de réguler leurs relations sociales, et participent ainsi à transnationaliser les normes de genre et les dispositifs de surveillance en vigueur dans les communautés d'origine $^{30}$. Toutefois, s'ils contestent rarement les assignations de genre en Indonésie et les formes de stratification sociale qui s'y attachent, beaucoup d'entre eux développent cependant un rapport ambivalent à d'autres formes d'inégalités. Ainsi, la distribution inégale du prestige social entre les générations et/ou au regard du lignage, au village, est de plus en plus mal vécue.

L'histoire de Budi, qui s'est aventuré sur la scène de la politique communale au retour de Corée du Sud, investissant le capital cumulé pour présenter sa candidature lors des élections municipales, est révélatrice de ces tensions : éduqué, formé comme professeur de religion, il est dépourvu de la profondeur lignagère qui soutient l'émergence des dirigeants sur la scène politique locale. A cette politique élitiste, fondée sur les formes traditionnelles de prestige social, il a tenté d'opposer sa légitimité religieuse et une éthique du travail appuyée sur son passé migrant :

«Le maire se préoccupe seulement... Il ne fait rien, il se contente de consigner les plaintes des habitants. Le reste, c'est de la corruption, seulement de la corruption. Aujourd'hui, du point de vue de la religion, c'est en fait la richesse des travailleurs migrants qui est licite - halal -, même si elle provient de l'étranger. Pourquoi? Parce que les travailleurs migrants ne

\footnotetext{
${ }^{28}$ Nina Glick Schiller, «Transnationality and the City », dans Gary Bridge et Sophie Watson (dir.), The New Blackwell Companion to the City, Chichester, Wiley-Blackwell, pp. 179-192.

${ }^{29}$ Une part significative des travailleuses migrantes vit en effet recluse dans les espaces de travail.

${ }^{30}$ Dans le contexte Thaïlandais on pourra se référer, sur ce thème, à : Kevin Bales, «Because she looks like a child », dans Barbara Ehrenreich et Arlie Russell Hochschild (dir.), Global woman: Nannies, maids and sex workers in the new economy, op. cit.
} 
manipulent pas d'argent sale. Leur richesse est le résultant de leur sueur. Les fonctionnaires... leur richesse est illicite - haram -. L'argent de l'étranger est plus propre! En comparaison de celui du régent - bupati - il est plus licite. »

Même s'il a échoué dans sa tentative électorale, défait par un notable, il esquisse les tenants d'une légitimité politique alternative, qui conteste la régulation traditionnelle du pouvoir.

L'irruption de ces revendications dissidentes s'explique par la discontinuité croissante entre les nouveaux processus de stratification sociale - transnationaux - et les structures communautaires en Indonésie. Là encore, la position des femmes dans les deux contextes est révélatrice[[B1].

Alors qu'elles subissent des dégradations statutaires au sein des sociétés d'origine, les femmes jouissent de meilleures positions dans les espaces transnationaux : d'abord par un pure effet démographique lié au volume supérieur des migrations féminines ${ }^{31}$; ensuite, parce que leur plus forte présence sur les routes migratoires les amène à accumuler des « savoir-circuler » et des compétences migratoires supérieures aux hommes ; parce qu'aussi, dans l'ensemble, elles ont accès à des destinations plus variées, où les possibilités d'accumulation économique sont les plus fortes, comme à Hong Kong et Taiwan, du fait notamment de contextes politiques et législatifs plus libéraux à l'égard des migrants ${ }^{32}$; enfin, parce que leur marginalisation symbolique au sein de l'ordre communautaire, au pays, tend à rendre les expériences migratoires plus gratifiantes pour elles, par comparaison. A raison de quoi elles tendent à développer des engagements transnationaux plus forts. En retour, la possibilité d'accéder à des positions sociales rehaussées, dans ces processus de stratification sociale «délocalisés », participe à renforcer leur inscription dans les espaces transnationaux et à affadir l'attachement aux espaces d'origine, en Indonésie.

Plus spécifiquement, alors que les assignations de genre et générationnelles persistent au village $^{33}$, elles sont profondément altérées dans les espaces transnationaux, dans la mesure surtout où les femmes et les jeunes forment l'essentiel des nouveaux contingents de migrants alors que la responsabilité de la subsistance familiale se transmet des parents aux enfants, dans un redéploiement contemporain de la circulation des obligations au sein de la famille nucléaire.

\footnotetext{
${ }^{31}$ En1996, les femmes indonésiennes représentaient 56\% des migrations de travail indonésiennes, alors qu'en 2008 ce ratio atteignait $78 \%$ (BIT 2010).

${ }^{32}$ Les différents pays gèrent les migrations de travail en appui sur une politique de quotas qui trie les migrants en fonction du genre et de la nationalité. Certaines destinations et certaines occupations sont ainsi interdites aux hommes.

${ }^{33} \mathrm{Ce}$ phénomène est bien documenté également dans le cas philippin. Cf. Gibson, Law, et McKay, «Beyond Heroes and Victims », International Feminist Journal of Politics, 3(3), 2001.
} 
D'un certain point de vue, les ordres sociaux apparaissent ainsi quasiment inversés : alors que les édiles continuent à jouir d'un fort statut social, localement, leur sédentarité les marginalise dans les espaces transnationaux. Les maisons qui marquaient leur affluence paraissent aujourd'hui insignifiantes, quand les flux financiers générés par la migration sont investis dans le foncier et l'habitat: de grandes demeures à l'architecture tapageuse sortent de terre, d'une valeur excédant parfois les 500 millions de rupiah ${ }^{34}$, surtout depuis que les migrations se sont ouvertes vers Taiwan, Hong Kong et la Corée du Sud, destinations les plus rémunératrices.

Par conséquent les circulations transnationales ont contribué à pluraliser les normes et les valeurs disponibles pour faire sens de l'expérience villageoise et des « épreuves » migratoires, en mettant en regard des positions souvent intraduisible dans l'ordre local et l'ordre transnational. Cette nouvelle pluralité normative éclaire partiellement les « exits » féminins : si certaines opposent le refus d'un retour à l'immobilité, s'engagent dans des cycles migratoires répétés et développent, ce faisant, des formes d'existence transnationales, c'est non seulement parce que les carcans collectifs leur paraissent désormais rédhibitoires (au village), mais aussi parce qu'elles accèdent ailleurs à des formes de rétribution symbolique qui leur permettent de jouir d'un statut social rehaussé, hors de la communauté ${ }^{35}$. Certaines femmes, parties il y a plus de vingt-cinq ans, ne sont jamais revenues.

En dépit de ces «turbulences », les processus de reproduction des structures communautaires résistent : en effet, si la migration affecte la vie commune, les expériences transnationales font réciproquement l'objet d'une reformulation en des termes compatibles à l'ordre hérité. On a alors moins affaire à la dissociation entre un «ordre nomade » émergent et un «ordre sédentaire $»^{36}$ institué qu'à un travail d'alignement qui tisse des continuités contre les effets disjonctifs des nouvelles migrations internationales. Par exemple, les pratiques propitiatoires qui ponctuent traditionnellement la vie communautaire, à Java, sont aujourd'hui convoquées pour protéger les migrants ; des repas rituels - selametan - sont ainsi organisés aux moments des départs et pour accompagner les retours, marquant symboliquement la sortie du collectif et la réintégration au sein de la communauté et assurant, ce faisant, la pérennité du fait communautaire face aux risques d'éclatement portés par la dispersion et l'hétérogénéité des expériences individuelles.

\footnotetext{
${ }^{34}$ Environ trente-cinq-mille euros.

${ }^{35}$ Bastide, «Habiter le transnational: Politiques de l'espace, travail globalisé et subjectivités entre Java, Kuala Lumpur et Singapour », thèse pour le doctorat de sociologie, Lyon, ENS de Lyon, 2011.

${ }^{36}$ Tarrius et Missaoui, Les nouveaux cosmopolitismes: Mobilités, identités, territoires, op. cit.
} 
Plutôt que la discontinuité tranchante que les migrations imposaient autrefois sur la vie communale, les trajectoires transnationales ont ainsi été construites comme une sorte de « rite de passage $»^{37}$, un espace-temps liminaire à l'intérieur d'un cycle de vie ordonné. Cependant, c'est l'idée même de ce retour à l'ordre, au terme de l'intermède migratoire, qui semble aujourd'hui mise en cause. Pour faire sens de ces phénomènes, un détour théorique s'impose.

\section{Le transnational et le local}

Parce qu'elles en grippent les rouages, les « épreuves » transnationales mettent en évidence les processus de reproduction de la communauté villageoise. Elles obligent ainsi à écarter les approches organicistes du fait communautaire en montrant que la persistance du collectif, loin de constituer un fait naturel, suppose un travail social, constant et obstiné, de délimitation, de réaffirmation et de redéfinition du commun qui le cimente ${ }^{38}$.

Au fond, ces transnationalismes contrastent une propriété empirique des collectifs sociaux, soit l'excès irréductible des réseaux relationnels et des pratiques par rapport aux unités sociales réputées les contenir. Ce constat a inspiré les propositions les plus fortes dans le champ de l'ethnographie multisite : dans le sillage des théories de l'acteur-réseau ${ }^{39}$ et des approches empiristes des phénomènes de globalisation ${ }^{40}$, elles suggèrent que l'extension des réseaux relationnels et des pratiques ${ }^{41}$ invalident toute prise de perspective holiste sur le social, qu'elle se rapporte aux micro-communautés de l'anthropologie classique, conçues comme entités closes et autosuffisantes, ou du «nationalisme » et de l'« ethnicité méthodologique ${ }^{42}$ qui tendent à naturaliser l'Etat-nation ou l'ethnos. Cette prémisse oblige à penser des dispositifs ethnographiques capables de prendre en charge les ramifications empiriques des phénomènes

\footnotetext{
${ }^{37}$ Arnold VAn Gennep, Les rites de passage: étude systématique des rites, Paris, Picard, 1991.Victor Turner, The ritual process : structure and anti-structure, New Brunswick, Aldine Transaction, 2008.

${ }^{38}$ Arjun Appadurai, Après le colonialisme: les conséquences culturelles de la globalisation, Paris, Payot, 2005 ; Rogers Brubaker, Ethnicity without groups, Harvard University Press, 2004.

${ }^{39}$ Bruno Latour, Changer de société, refaire de la sociologie, Paris, la Découverte, 2007.

${ }^{40}$ Sur ce thème on lira par exemple : Aihwa Ong et Stephen J. Collier, Global assemblages : technology, politics, and ethics as anthropological problems, Wiley-Blackwell, 2005; Anna L. Tsing, Friction: an ethnography of global connection, Princeton, Princeton University Press, 2005; Saskia Sassen, La globalisation: une sociologie, Paris, Gallimard, 2009.

${ }^{41}$ Mark-Anthony Falzon (dir.), Multi-sited ethnography: theory, praxis and locality in contemporary research, op. cit.

${ }^{42}$ Beck, Pouvoir et contre-pouvoir à l'ère de la mondialisation, Flammarion, 2009; Andreas Wimmer et Nina Glick-Schiller, «Methodological Nationalism, the Social Sciences, and the Study of Migration », International Migration Review, 37(3), 2003, p.576-610; Nina Glick Schiller, «Beyond Methodological Ethnicity», Willy Brandt Series of Working Papers in International Migration and Ethnic Relation, 2008.
} 
sociaux et amène régulièrement le chercheur à désenclaver sa pratique pour la porter en plusieurs lieux ${ }^{43}$.

Ces propositions permettent d'échapper au "piège identitaire $»^{44}$. Entre autres vertus, elles préviennent contre la mobilisation a-réflexive des catégories identitaires maniées par les acteurs sociaux pour se représenter le monde, et pour présenter leur monde au chercheur. L'ethnographie des expériences migrantes à Singapour et Kuala Lumpur éclaire pleinement ce point : dans les deux métropoles, les acteurs développent des pratiques et des modes de vie souvent transgressifs des normes en vigueur dans les collectifs d'origine. Pour les plus jeunes, encore célibataires, l'expérience métropolitaine est souvent l'occasion d'expérimenter des pratiques de soi et des modes de vie inédits : des femmes nouent par exemple des relations amicales, amoureuses ou sexuelles avec des compatriotes ou des nationaux, et évoluent souvent à la croisée de cercles d'interconnaissance pluriels. Certaines multiplient aussi les activités : à Singapour, des travailleuses s'engagent par exemple dans des mobilisations collectives, au sein d'ONG locales ou dans un groupe d'entraide créé par des migrantes indonésiennes ; d'autres suivent des cours et des formations auprès d'organismes publiques et d'ONG, ou développent des activités dans le domaine de la 《petite production urbaine ${ }^{45}$. Ces pratiques et les processus d'individuation liés aux « épreuves sociales » dont elles sont porteuses accentuent la prise de distance face aux positions et aux rôles sociaux auxquels elles sont assignées, en Indonésie. Si l'étiolement des retours indique alors un désajustement de plus en plus prononcé avec les réquisits de la vie collective «au pays », cette expérience suscite rarement la formulation d'une critique qui mettrait en cause les assignations sociales dont ils sont porteurs et qui rendent, précisément, une réinstallation de moins en moins envisageable : ces femmes persistent dans l'expression vivace de loyautés culturelles vis-à-vis de ces appartenances, vécues sur un mode naturalisé, et les formes réglées de vie collective dont elles sont porteuses.

Les conceptions réticulaires du social développées par les praticiens de l'ethnographie multisite offrent un gain heuristique difficile à négliger. Entre autres choses elles permettent de sortir de la référence systématique à l'Etat Nation qui empêtre les théories du transnationalisme, dont l'objet existe, bien souvent, par contraste avec le fait national. En cela, elles prolongent et

\footnotetext{
43 George E. Marcus, «Multi-sited ethnography: Notes and Queries », dans Mark-Anthony Falzon (dir.), Multi-sited ethnography: theory, praxis and locality in contemporary research, Farnham, Burlington, Ashgate Publishing, pp. 181-196.

${ }^{44}$ Michel Agier, La condition cosmopolite: l'anthropologie à l'épreuve du piège identitaire, Paris, la Découverte, 2013.

${ }^{45}$ Roulleau-Berger, Le travail en friches, Ed. de l'Aube, 1999.
} 
dépassent leur paradigme ${ }^{46}$. La recension scrupuleuse des pratiques, sans référent politicoterritorial a priori permet de recouvrer la richesse empirique des chaines relationnelles, de mettre en évidence l'extension effective des pratiques et de désarmer ainsi les discours identitaires et les proclamations d'appartenance trop tranchées à des collectifs fermés. Les tensions propres à ces expériences féminines montrent qu'il faut cependant pouvoir traiter la propension des acteurs à se situer dans le monde par rapport à des référents identitaires substantiels. S'il est indispensable de reconnaître que les manifestations de loyautés culturelles sont, sinon démenties, au moins amendées par des pratiques dissonantes, il convient alors aussi de ne pas pécher par excès inverse : on ne peut ignorer leur capacité à structurer les perceptions des acteurs ni, par conséquent, sous-estimer leurs effets pratiques. Sur ce point, les propos de Bayu, à Kuala Lumpur offrent un vigoureux rappel :

" [Le Javanais] est exemplaire, grand frère. Oui... il ne se fâche pas facilement. Il y a un proverbe javanais qui dit "contrôle-toi »... «Délibères au préalable ». Il ne se fâche pas facilement. [...] il a l'habitude d'être paysan au village. Il est habitué. C'est différent avec les Madurais $^{47}$. Le Madurai c'est un idéologue. La politique... il est dur. Il s'adapte à son environnement. Lui, c'est...il est pêcheur, et c'est le sel. Le pêcheur, n'est-ce pas il... comme on dit il travaille sur la mer, il subit la chaleur... [...] il devient chaud. [...] Quand il se bat, qui c'est qui gagne? C'est celui qui gagne qui a raison. Celui qui meurt, c'est lui qui a tort. Bien que celui qui est mort il n'y ait aucune garantie qu'il ait eu tort ! [...] C'est le jugement de dieu. La loi de la jungle, oui! C'est ça! Mais celui qui peut... se mesurer à lui c'est le Florésien $^{48}$...en Malaysia. [...] Son principe à lui...à ce Florésien, c'est le suivant : "Tu manges du riz. Je mange du riz. Tu es humain, ton sang est rouge, moi aussi j'ai le sang rouge. Ok. Même je... je me fais poignarder ou que je me fais...si Dieu n'a pas décidé que c'était mon heure, je ne peux pas mourir». C'est comme ça avec le Florésien. »

Anna L. Tsing ${ }^{49}$, une anthropologue américaine, pose une approche qui permet de réduire ces tensions théoriques : pour elle, la théorie de l'acteur réseau critique avec raison le « holisme méthodologique » qui mine la pratique d'enquête. En sa radicalité, suggère-t-elle, elle conduit

\footnotetext{
${ }^{46}$ Pour une présentation synthétique des études transnationales, cf. Rainer Bauböck et Thomas Faist, Diaspora and Transnationalism: Concepts, Theories and Methods, Amsterdam, Amsterdam University Press, 2010; Steven Vertovec, Transnationalism, Oxon, New York, Routledge, 2009.

${ }^{47}$ Population de Madura, une île située au large de la côte nord de Java Est, vers Surabaya.

${ }^{48}$ Originaires de Nusa Tenggara Timur (NTT), petites îles de la Sonde, de l'île de Flores située entre Bali et le Timor, espace de transition entre les phénotypes sud-est asiatique et mélanésien, qui accentue la perception de la différence.

${ }^{49}$ A. L. Tsing, « Worlding the Matsutake Diaspora: Or, Can Actor-Network Theory Epxeriment With holism? », in Ton Otto et Nils Bubandt (dir.), Experiments in Holism: Theory and Practice in Contemporary Anthropology, Wiley-Blackwell, 2011.
} 
cependant à négliger le « holisme expérientiel $»^{50}$ développé par les acteurs sociaux pour se représenter le monde. Il conviendrait ainsi de combiner une approche scientifique des réseaux et des pratiques, libérée du «holisme méthodologique », tout en faisant voix au «holisme expérientiel » des acteurs sociaux, à condition cependant de le situer au bon endroit, non plus comme outil mais comme objet de l'analyse. Ces réflexions rappellent la nécessité qu'il y a à penser ensemble la fragmentation des pratiques et l'extension empirique des chaînes relationnelles, d'une part, et la tendance des acteurs à se représenter le monde à partir de catégories substantielles, d'autre part.

En distinguant entre voisinages et localité, Arjun Appadurai propose une approche conceptuelle qui permet précisément de répondre à ces inflexions et de penser le rapport entre repères identitaires (holisme expérientiel) et prolifération des relations et des pratiques, dans une démarche analytique qui porte explicitement sur la question des processus de formation et de spatialisation des collectifs.

Appadurai pose en ces termes le rapport entre voisinages et localité ${ }^{51}$ : un « voisinage » réfère à une formation sociale caractérisée par un ensemble de productions matérielles et immatérielles qui font ressource (et contexte) à la vie collective - relations économique, structures d'habitat, systèmes de parenté, histoires collectives, mythes, structures de réciprocité, etc. Le concept de localité, quant à lui, est débarrassé de sa dimension spatiale ; il est alors employé pour qualifier les « structures de sentiment » constituées dans ces voisinages, et qui sont au principe de la perception par les acteurs des identités collective ${ }^{52}$. La localité est ainsi à la foi une propriété de la vie locale et une idéologie communautaire. Ainsi pensée, elle permet de saisir les modalités et les processus d'institution d'un sujet local, à partir d'un voisinage dans lequel sont assurées les conditions de production et de reproduction d'un sentiment d'appartenance partagé.

Est ainsi préservée la distinction essentielle entre la matérialité des relations et des pratiques, et la réalité phénoménologique des processus d'identification. Mais on peut désormais spécifier la relation entre ces deux dimensions : si la «localité »s'enracine toujours dans un « voisinage », un « voisinage » ne fait pas forcément localité, c'est-à-dire qu'il ne donne pas toujours lieu à la production d'un sujet local, inscrit dans un collectif lié par des structures de

\footnotetext{
${ }^{50}$ Ton Otto et Nils Bubandt, «Introduction », dans Ton Otto et Nils Bubandt (dir.), Experiments in Holism: Theory and Practice in Contemporary Anthropology, op. cit. pp. 1-16.

${ }^{51}$ Arjun Appadurai, Après le colonialisme, op. cit. 257-284.

52 Arjun Appadurai, Après le colonialisme, op. cit., p. 257-258.
} 
sentiment. Ce faisant, Appadurai renverse les attendus du raisonnement anthropologique en montrant que la « localité » n'est pas un donné mais résulte d'un effort constant, incertain, sans cesse remis sur le métier, renégocié pour maintenir ses conditions de félicité à travers des circonstances changeantes.

On peut relire l'expérience locale, à Banyu Putih, à la lumière de ces arguments.

Avant les années 1980 l'essentiel des existences se déroulait au village. Cette fixité conférait leur force aux structures de la vie collective, qui facilitait la coïncidence entre un « voisinage » relativement circonscrit, fortement ancré, des «structures de sentiment » et des identifications collectives. Dès les années 1980 les migrations ont provoqué la dispersion du collectif. Comme on l'a vu, la dissémination des expériences a donné lieu à la production de savoirs, de savoirfaire et de savoir-être partagés, spécifiquement liés à la migration, à l'apparition de nouvelles formes relationnelles, à l'institutionnalisation progressive de liens et de pratiques transnationaux, producteurs de nouvelles «capacités à aspirer ${ }^{53}$. Ces nouvelles formes sociales composent aujourd'hui autant de « voisinages », dispersés entre l'Indonésie, l'Asie de l'Est et du Sud-Est et les pays du golfe persique. Les migrations assemblent ainsi des réseaux longs d'affiliations pluri-situées, producteurs de nouvelles « épreuves sociales », où se nouent de nouvelles loyautés et de nouvelles appartenances, ancrées au sein de collectifs culturellement hétérogènes, plus ou moins éphémères et géographiquement dispersés et où, surtout, se déploie désormais une part croissante des existences individuelles. Des «structures de sentiment » se composent, liées à ces expériences, qui attachent les migrants aux lieux de migration; des translocalités apparaissent, générées dans des voisinages transnationaux, productrices d'affiliations et d'identifications discrètes, où le village vaut désormais comme un lieu singulier dans la chaine des lieux pratiqués. Parallèlement, la reproduction matérielle du village comme voisinage dépend de plus en plus des boucles transnationales, qui répondent à des modes de vie de plus en plus monétarisés et onéreux, liés entre autres choses à l'appétence croissance pour les biens de consommation, aux coûts d'entretien de l'investissement foncier, et à la scolarisation prolongée des enfants.

Du point de vue de la société villageoise, ces « voisinages » transnationaux sont d'autant plus corrosifs qu'ils se construisent à partir de parcours individuels marqués par les contingences de tracés spatiaux fortement différenciés, frayés par des migrants qui combinent les étapes et

\footnotetext{
${ }^{53}$ Pour Appadurai, la capacité d'aspiration, comme mode d'appropriation du futur, n'est pas également distribuée : la répartition de cette compétence forme une modalité spécifique d'inégalité sociale. Arjun Appadurai, The future as cultural fact : Essays on the global condition, London, New York, Verso, 2013, p.179-197.
} 
les lieux de manière toujours singulière. L'expérience partagée, au village, qui englobait les existences individuelle reflue et compose désormais une facette circonscrite au sein de biographies pluri-situées.

\section{Localité, translocalités et rapports de pouvoir}

En brouillant les frontières et en inscrivant durablement le village dans un faisceau de relations, de pratiques et d'affiliations de plus en plus ample, la migration altère ainsi les processus de reproduction de la « localité », autrefois facilités par une forte coïncidence entre territoire et voisinages, entre un sol, un collectif relativement sédentaire, et un régime d'identification partagé. La pluralisation des voisinages, dans lesquels circulent des pratiques et des normes discontinues et contrastées, met à nu ces mécanismes auparavant largement vécus sur le mode de l'évidence. Dans ces circonstances, les hiérarchies sociales sont de moins en moins perçues comme allant de soi et l'ordre social se recompose sous l'effet de sa re-localisation dans ces nouveaux « voisinages ».

Les formes sociales sont en effet fortement altérées par ces redéploiements : L'ancrage local s'insère désormais dans la longue chaîne des «épreuves » caractéristiques des circulations migratoires. L'agriculture, qui valait auparavant comme moyen de subsistance, est par exemple bouleversée par son enchâssement dans ces nouveaux répertoires d'expériences. Cette pierre angulaire de la société locale, qui contribuait à la reproduction matérielle du collectif et à la reproduction symbolique des hiérarchies sociales ${ }^{54}$, voit ces deux fonctions s'épuiser : d'une part, l'investissement des capitaux issus des migrations a participé à découpler le capital foncier du capital symbolique (par sa «vulgarisation» du fait des acquisitions par la « roture ») ; d'autre part les migrations, par le volume des capitaux qu'elles génèrent, ont progressivement marginalisé la place de l'agriculture dans l'économie villageoise. Aujourd'hui les pratiques agricoles se transforment et contribuent, surtout, à re-socialiser les migrants aux rites et aux rythmes de la vie commune, après parfois de longs séjours dans des métropoles trépidantes : le retour à la terre, souvent sans nécessité économique, permet de refroidir $^{55}$ le nomade, de le resocialiser. Plus largement, on a vu que les rituels sont mobilisés pour accompagner la sortie et la réintégration des migrants dans l'ordre villageois, c'est-à-dire pour assurer la reproduction d'un sujet local, contre l'effet érosif des boucles transnationales.

\footnotetext{
${ }^{54}$ Ann L. Stoler, « rice harvesting in Kali Loro », American Ethnologist, 4(4), 1977, 678-698.

${ }^{55}$ Cette expression est empruntée aux acteurs.
} 
Ces mutations, on l'a dit, ne vont pas sans poser problème. La tentative politique de Budi, montre assez qu'elles participent à ouvrir de nouveaux espaces de controverses où des pratiques innovantes, liées aux expériences migratoires, s'affrontent à des résistances et suscitent des replis identitaires dansl'ordre traditionnel.

Ainsi, le maintien de plus en plus anxieux des évènements cérémoniels - repas propitiatoires, mariages, circoncisions, veillées mortuaires, travail collectif bénévole (kerja bakti) ou pratiques d'entraide (gotong royong), etc. - suggère à la fois l'omniprésence du sentiment d'un monde en danger et l'investissement collectif dans la reproduction d'une expérience sociale partagée. Le maintien d'une vie associative intense souligne encore l'engagement collectif dans le maintien de l'ordre commun. Les petites organisations communales, qui maillent la vie locale dans l'archipel ${ }^{56}$, sont en effet agrégées en fonction de critères de genre, de classes et de génération. La réitération de ces affiliations et de ces pratiques collectives et le maintien de la vie rituelle participent ainsi à re-clarifier et renforcer les stratifications sociale héritées. Ce qui semble en jeu ici, c'est la définition et la réaffirmation des frontières entre localité et translocalités, face à l'éparpillement progressif du collectif en ses nouveaux « voisinages ». Cet effort constant de re-clarification, pour ainsi dire, - clarification de la nature et des formes appropriées du lien social, de l'identité commune et du territoire collectif - répond à l'anxiété profonde suscitée par les transformations rapides des structures sociales : ce travail social, qui engage tout un chacun, porte en définitive sur la réaffirmation de plus en plus problématique de la communauté comme totalité sociale, contre sa dissolution transnationale.

A Banyu Putih ces processus de reproduction se vivaient moins auparavant comme pratique sociale ou politique que sur le mode de l'évidence, comme manière d'être au monde. La force des routines et des « rites d'interaction $\|^{57}$ maintenait un sentiment d'appartenance collectif, le sens culturel vivace d'un «faire société ». Le collectif était moins perçu alors comme un construit social fragile que comme un ordre naturel, doté de la robustesse du réel. Certes, la vie collective n'était ni consensuelle ni dénuée de conflits sociaux. L'avancer serait s'enfermer dans un «rousseauisme » naïf sur le plan conceptuel et empiriquement intenable. Cependant, ces conflits ne visaient pas l'ordre social des positions, dans un contexte, à Java, où l'aptitude

\footnotetext{
${ }^{56}$ Citons, par exemple, karang taruna pour la jeunesse, le linsmas (autrefois hansip), formation paramilitaire, ou les PKBI (Perkumpulan Keluarga Berencana Indonesia), groupes de planning familial féminin ou encore les organisations religieuses, comme la branche locale de la Nadhlatul Ulama, la première organisation musulmane d'Indonésie, ou les cercles d'étude du coran - kelompok pengajian -. La liste est loin d'être exhaustive.

${ }^{57}$ Erving Goffman, Les rites d'interaction, Paris, Éd. de Minuit, 1974.
} 
de chacun à connaître et répondre aux attendus de sa juste position dans l'ordre social est fortement valorisée et définit les conditions culturelles de la « vie bonne $»^{58}$.

Si la reproduction de l'expérience locale engage le collectif en son entier, il est alors très significatif de constater que les acteurs les plus investis dans la poursuite des activités rituelles et associatives et les plus affectés par l'érosion des modes de vie hérités fassent généralement partie de la petite notabilité villageoise : Kamardi, commerçant prospère, déplore ainsi avec dérision et quelque amertume la transformation de Banyu Putih en un «village arabe » - desa arab -, et dénonce l'altération des rôles sexués : «Ça alors, les hommes maternent! 》- « Kok, lelaki momong!». L'activité publique du secrétaire de mairie (un raden ${ }^{59}$ de la petite aristocratie, figure politique la plus influente du village) et de son épouse, qui jouent un rôle central dans le maintien de la vie rituelle et associative, démontre encore l'importance des hiérarchies sociales pour la répartition des rôles dans le contexte de ces luttes reproductives : elle semble en effet largement déterminée par les positions occupées au sein des stratifications sociales héritées. Les acteurs parés du plus fort prestige et dotés du pouvoir politique sont généralement les gardiens les plus zélés des modes d'existence hérités. Les fractions les plus marginalisées de la population, dans l'ordre social et symbolique (les femmes, les jeunes et les déshérités) tendent au contraire à en renégocier les termes, sinon de manière revendicative, au moins en pratique, parfois par des départs définitifs.

Deux choses valent alors d'être relevées :

En premier lieu, les formes culturelles héritées, drossées aujourd'hui contre ces nouvelles «transnationalités ${ }^{60}$, perdent progressivement leur qualité de « seconde nature » : ce qui se confondait auparavant avec l'« en soi » du monde, pour ainsi dire (les hiérarchies sociales, les rituels d'interactions, etc.), se révèle comme matière culturelle, c'est-à-dire située et relative (plutôt que substantielle). Cette transformation compromet le caractère hégémonique des formes culturelles établies : alors qu'elles travaillaient les pratiques et les perceptions de l'intérieur, elles sont aujourd'hui progressivement projetées sur le plan du débat et de la controverse, à la fois sur le plan local et sur les lieux de migration.

Dans ce nouvel espace politique, des acteurs brandissent la valeur de l'héritage culturel et développent un discours déploratif sur la perte d'identité et l'évanouissement des traditions,

\footnotetext{
${ }^{58}$ Denys Lombard, Le carrefour javanais : essai d'histoire globale. I, Les limites de l'occidentalisation, vol 3, Paris, Éd. de l'ÉHESS, 2004; Benedict O. G. Anderson, Language and Power: Exploring Political Cultures in Indonesia, Singapore, Equinox Publishing, 2006.

${ }^{59}$ Titre nobiliaire.

${ }^{60}$ Glick Schiller, « Transnationality and the City », art. cité.
} 
comme le maire ou son secrétaire de mairie, tandis que d'autres, aujourd'hui, réinvestissent et retravaillent les formes culturelles en puisant dans des répertoires normatifs et des registres de valeurs plus congruents avec les épreuves transnationales qui forment l'ordinaire des expériences sociales contemporaines. Ainsi, alors que les relations de genre et les pratiques sexuelles développées par certains migrants à l'étranger sont fortement stigmatisées dans les communautés d'origine, elles rencontrent des formes liminales de reconnaissance et de légitimité dans des «espaces intermédiaires ${ }^{61}$ transnationaux, au sein de collectifs souvent pluri-culturels, mêlant circulants et sédentaires à Kuala Lumpur, Singapour, Hong Kong, Séoul ou Taipeh.

En second lieu, alors que les formes culturelles établies perdent de leur efficace, parce qu'elles échouent à régler des pratiques nouvelles, leur complicité avec les structures de pouvoir est exposée $^{62}$ : Depuis Gramsci, on sait en effet que la dimension culturelle est essentielle à la structuration, la stabilisation, la légitimation et la reproduction des rapports de pouvoir ${ }^{63}$. Nulle surprise alors à ce que les plus ardents défenseurs de l'authenticité, qui conjurent la légitimité des traditions, occupent des positions centrales dans l'ordre symbolique du prestige social : dans la mesure où les normes et les valeurs héritées contribuent à reconduire les structures sociales et les rapports de pouvoir, le délitement des institutions culturelles les menace en leur personne sociale. C'est d'autant plus vrai que les notables occupent des positions marginales dans les voisinages transnationaux.

Si ces phénomènes témoignent des profondes mutations à l'œuvre au village, l'efficacité idéologique des positions conservatrices dans ces luttes pour la reproduction ne peut cependant être éludée. En effet, si on excepte le crédo politique de Budi, on constate l'absence quasitotale de discours d'opposition aux conservatismes localistes, que ce soit au village ou dans les espaces de migration. Au village, les acteurs expriment généralement un engagement durable et un attachement profond aux modes d'existence traditionnels, en dépit de leur engagement dans des pratiques qui les altèrent radicalement. A l'étranger, l'hiatus est plus manifeste encore : alors que les travailleurs migrants - surtout les femmes - développent des pratiques et des pratiques de soi souvent très transgressives, elles sont très rarement transcrites dans des discours critiques, alors même que l'ordre social, dans les communautés d'origine, réprime sévèrement ces identités émergentes.

\footnotetext{
${ }^{61}$ Laurence Roulleau-Berger, « La production d'espaces intermédiaires », Hermès, 36, 2003.

${ }^{62}$ Aihwa Ong, Flexible citizenship: The cultural logics of transnationality, Durham, Londres, Duke University Press, 1999.

${ }^{63}$ Antonio Gramsci, Selections from the Prison Notebooks of Antonio Gramsci, New York, International Publishers, 1971.
} 
Les théories de l'hégémonie expliquent parfaitement cette apparente contradiction, en rappelant le rôle des formes culturelles dans l'organisation des rapports de pouvoir. On sait aussi, cependant, que l'élasticité du rapport entre discours est pratique connaît des limites ${ }^{64}$. A ce titre, l'aventure politique de Budi pointe l'émergence de contre-discours, contestataires, plus congruents avec la consolidation des nouvelles translocalités, les expériences et les pratiques qui s'y enchâssent. A Hong Kong, des associations de travailleurs indonésiens liés à des syndicats philippins et des associations en Indonésie s'approprient une critique marxiste des rapports de classe et une critique féministe des rapports de genre pour attaquer les pays impliqués dans le commerce des migrants. A Kuala Lumpur et Singapour l'écart croissant entre des modes de vie transgressifs, des formes de subjectivation nouvelle et la réaffirmation des loyautés traditionnelles dès qu'on suscite un discours sur les espaces d'origine se résout souvent par des exits prolongés, qui permettent de gérer ses coûts moraux ${ }^{65}$.

Dans ce contexte, la culture «patrimonialisée » joue un rôle d'auxiliaire dans une «politique de l'identité ${ }^{66}$, qui se comprend comme résistance de la « localité » face à son intégration et à son affadissement à l'intérieur de nouvelles formations socio-spatiales. Ce faisant, la répartition sociale des positions dans le cadre de ces luttes et de ces résistances met en évidence des rapports de pouvoir auparavant opacifiés par les routines sociales. Et les normes et valeurs héritées et consensuelles, qui posaient auparavant les cadres du vivre en commun, font aujourd'hui disensus : alors qu'elles définissaient les termes et les cadres du débat public, elles deviennent, progressivement, l'objet de la controverse politique.

\section{Conclusion}

Ces conflits très locaux s'inscrivent aujourd'hui dans des reconfigurations plus amples de l'espace politique autour de la question migratoire, au moment où elle émerge comme une question sociale majeure au niveau régional et suscite de vives controverses entre les états, les institutions internationales, les ONG et au sein des sociétés nationales. Les débats de plus en plus intenses autour du commerce de la main d'œuvre suscitent des transformations rapides des politiques de placement et des «économies morales » autour de ces questions. En 1995

\footnotetext{
${ }^{64}$ Sur le rapport entre institution de la réalité et changement social, on renvoie à: Luc Boltanski, De la critique : Précis de sociologie de l'émancipation, Paris, Gallimard, 2009.

${ }^{65}$ Loïs Bastide (A paraître), « «Migrer, être affecté » Emotions et expériences spatiales entre Java, Kuala Lumpur et Singapour », REMI, 2014.

${ }^{66}$ Jonathan Hill et Thomas Wilson, « Identity Politics and the Politics of Identities », Identities: Global Studies in Culture and Power, 10(1), 2003, p.1-8.
} 
l'exécution de Flor Contemplacion, une travailleuse domestique philippine à Singapour provoquait un tel outrage dans le pays que le gouvernement philippin se voyait forcé à réorganiser sa politique de placement de fond en comble.

Les transformations à l'œuvre, à Banyu Putih, s'inscrivent ainsi dans un cadre plus large : l'émergence de nouvelles « économies morales » est connectée à la construction conflictuelle de la question migratoire à l'échelle nationale et régionale, dans la mesure au moins où les controverses qui s'y rapportent pluralisent les discours disponibles pour construire le sens de l'expérience circulatoire, au village, et renforcer des positions contestataires. Des ONG et des organisations internationales comme l'Organisation International des Migrations (IOM) ou le Bureau International Du Travail (BIT) portent en effet des discours de valorisation des migrations de travail et reformulent les expériences transnationales en des termes positifs. Le gouvernement indonésien, de plus en plus dépendant de la rente migratoire, cherche aussi à imposer un discours mélioratif pour susciter les vocations et affirmer sa solidarité à l'égard de ses citoyens expatriés, comme l'illustre la nomenclature officielle qui présente les migrants comme des « héros des devises ${ }^{67}$ (pahlawan devisa) en référence à leur rôle cardinal dans l'économie nationale. Sous la pression croissante de la société indonésienne, le gouvernement est aussi amené aujourd'hui à revoir ses relations avec les pays recruteurs, à mesure que les cas de maltraitance se multiplient et suscitent une indignation politiquement coûteuse au sein de la société indonésienne.

Faire l'ethnographie des processus de (re)production de la localité, dans ce contexte, est rien moins qu'anecdotique: une manière de se donner un point d'entrée empirique sur des phénomènes qui emportent les sociétés à l'échelle régionale et continentale, interrogeant leurs ancrages sociaux, politiques, culturels et territoriaux.

\footnotetext{
${ }^{67}$ Terme emprunté aux Philippines.
} 